\title{
On the Connected Components of the Space of Line Transversals to a Family of Convex Sets*
}

\author{
J. E. Goodman, ${ }^{1}$ R. Pollack, ${ }^{2}$ and R. Wenger ${ }^{3}$ \\ ${ }^{1}$ City College, City University of New York, \\ New York, NY 10031, USA \\ jegcc@cunyvm.cuny.edu \\ ${ }^{2}$ Courant Institute of Mathematical Sciences, New York University, \\ New York, NY 10012, USA \\ pollack@geometry.nyu.edu \\ ${ }^{3}$ Ohio State University, Columbus, OH 43210, USA \\ wenger@cis.ohio-state-.edu
}

\begin{abstract}
Let $\mathscr{L}$ be the space of line transversals to a finite family of pairwise disjoint compact convex sets in $\mathbb{R}^{3}$. We prove that each connected component of $\mathscr{L}$ can itself be represented as the space of transversals to some finite family of pairwise disjoint compact convex sets.
\end{abstract}

\section{Introduction}

Let $\mathscr{A}$ be a family of convex sets in $\mathbb{R}^{d}$. A $k$-transversal for $\mathscr{A}$ is a $k$-flat, an affine subspace of dimension $k$, that intersects every member of $\mathscr{H}$. (For basic facts about transversal theory, see [1] or [3].) Let $\mathscr{A}^{*}$ be the set of all $k$-transversals of $\mathscr{A}$, considered as a subspace of the affine Grassmannian $\mathbf{G}_{k, d}^{\prime}$ of all $k$-flats in $\mathbb{R}^{d}$. We are interested in studying the connected components of this space $\mathscr{B}^{*}$ and, in particular, in the question of whether each of these connected components can be represented as the space of $k$-transversals of some other family $\mathscr{B}$ of convex sets.

* The research of J. E. Goodman was supported in part by NSF Grant DMS91-22065 and by NSA Grant MDA904-92-H-3069. R. Pollack's research was supported in part by NSF Grant CCR91-22103 and by NSA Grant MDA904-92-H-3075. The research of $\mathrm{R}$. Wenger was supported in part by NSA Grant MDA904-93-H-3026 and by the NSF Regional Geometry Institute (Smith College, July 1993) Grant DMS90-13220. 
This question was posed in [2], where it was shown that a convexity structure can be defined on $\mathbf{G}_{k, d}^{\prime}$ in which the convex sets are precisely the sets of $k$-transversals to families of convex point sets in $\mathbb{R}^{d}$. This convexity structure coincides with the usual one on $\mathbb{R}^{d}$ if $k=0$, and retains many of its properties for $k>0$. In particular, the convex hull of a family $\mathscr{F}$ of $k$-flats can be defined by means of a duality operator, *, between sets of $k$-flats and families of convex point sets, as follows. If $\mathscr{F}$ is a family of $k$-flats and $\mathscr{A}$ is a family of convex point sets in $\mathbb{R}^{d}$, let $\mathscr{F}^{*}$ be the family of convex point sets meeting every member of $\mathscr{F}$ and let $\mathscr{A}^{*}$ be the family of $k$-flats meeting every member of $\mathscr{A}$. Then conv $(\mathscr{F})$, the convex hull of $\mathscr{F}$, is simply $\mathscr{F}^{* *}$. A family $\mathscr{F}$ of $k$-flats is convex if conv $(\mathscr{F})=\mathscr{F}$. It is easily seen that $\mathscr{A}^{*}$ is convex for any family $\mathscr{A}$ of convex point sets.

One can equally well obtain the convex hull as follows. A family $\mathscr{F}$ of $k$-flats is said to surround a $k$-flat $f$ if there is some $j$-flat $g$ containing $f$ such that every $(j-1)$-flat $h$ containing $f$ and lying in $g$ strictly separates two $k$-flats of $\mathscr{F}$ lying in $g$; i.e., $h$ cannot "escape to infinity" within $g$ without containing a member of $\mathscr{F}$ after moving some positive distance. (If $j=k$ we interpret this condition as meaning that $f \in \mathscr{F}$.) A family $\mathscr{F}$ of lines in $\mathbb{R}^{3}$, for example, surrounds a line $f$ if either

(i) $f \in \mathscr{F}$,

(ii) $f$ lies strictly between two lines of $\mathscr{F}$ with all three contained in a plane $g$, or

(iii) each plane $h$ through $f$ lies strictly between two lines of $\mathscr{F}$ parallel to $h$.

The family of all $k$-flats surrounded by $\mathscr{F}$ turns out to be exactly the convex hull of $\mathscr{F}$ [2]. Notice that this characterization of the convex hull applies equally well to 0 -flats (or points) in $\mathbb{R}^{d}$, where it agrees with the standard one for point sets. (For a complete discussion of convexity on the affine Grassmannian, see [2].)

If $\mathscr{F}$ is a convex set of $k$-flats in $\mathbb{R}^{d}$, then $\mathscr{F}=\mathscr{A}^{*}$ for some family $\mathscr{A}$ of convex point sets in $\mathbb{R}^{d} ; \mathscr{F}$ is then said to be presented by $\mathscr{A}$. A convex set $\mathscr{F}$ may be presented by many different families of convex point sets.

A convex set of $k$-flats need not be connected for $k>0$. For instance, a finite family of mutually skew lines in $\mathbb{R}^{3}$ is convex, by the "surrounding" criterion. Problem 7.2 of [2] asks whether the connected components of a convex set of $k$-flats are necessarily convex. In general the answer turns out to be negative, as we show by exhibiting an example of a convex family of lines in $\mathbb{R}^{3}$ that has a nonconvex connected component. If, however, a convex set of $k$-flats in $\mathbb{R}^{d}$ is presented by some finite family of suitably separated compact convex point sets, then we conjecture that the connected components of $\mathscr{F}$ will themselves be convex. We prove this conjecture for the case of a convex set of lines in $\mathbb{R}^{3}$ presented by a finite family of pairwise disjoint compact convex point sets; this is the main result of this paper.

\section{A Nonconvex Connected Component}

In this section we exhibit a connected set $\mathscr{L}$ of lines in $\mathbb{R}^{3}$ whose convex hull has two arcwise connected components. One of these components must contain $\mathscr{L}$, so it cannot be convex. 
Start with the set of all the tangents to a unit circle centered at $O$ in the $(x, y)$-plane. Let $\alpha$ be an angle irrational with respect to $\pi$ and for each $n=1$, $2, \ldots$ translate the pair of lines tangent at $(\cos n \alpha, \sin n \alpha)$ and $(-\cos n \alpha,-\sin n \alpha)$ toward $O$ until they are each at distance $1 / n$ from it. For each direction $\theta$, $0 \leq \theta<\pi$, we now have a pair of parallel lines around $O$ in direction $\theta$; fill in all the lines between these two parallels in each direction $\theta$, getting a strip $S_{\theta}$. It follows from the irrationality of $\alpha$ that arbitrarily close to each direction there are arbitrarily narrow strips.

Finally, for each $\theta, 0 \leq \theta<\pi$, translate this strip $S_{\theta}$ upward to a strip $T_{\theta}$ at height $z=\theta$. This gives a "spiral staircase" $\mathscr{L}$ having the $z$-axis as an axis of symmetry, closed at the height $z=0$ and open at the height $z=\pi$.

Each line in a strip $T_{\theta}$ is connected to the member of $T_{\theta}$ passing through the $z$-axis. Since the latter form a connected family for $\theta \in[0, \pi), \mathscr{L}$ is connected. We claim that the convex hull of $\mathscr{L}$ is $\mathscr{L} \cup\{z$-axis $\}$, so that conv $(\mathscr{L})$ has two components, $\mathscr{L}$ and the $z$-axis. We show this by using the "surrounding" criterion above.

The fact that the $z$-axis $\in \operatorname{conv}(\mathscr{L})$ is seen by considering any plane through the $z$-axis; since each $T_{\theta}$ has positive width this plane will be trapped by lines in $\mathscr{C}$ strictly parallel to it, so that the $z$-axis is surrounded by $\mathscr{L}$.

It remains only to show that no other line $l \notin \mathscr{L}$ lies in conv $(\mathscr{L})$. If $l$ is parallel to the $(x, y)$-plane, any plane $\Pi$ through $l$ not itself parallel to the $(x, y)$-plane has lines of only a single strip $T_{\theta}$ parallel to itself, with $T_{\theta}$ depending only on the line $l$, so that by rotating $\Pi$ around $l$ we can find a position in which $\Pi$ lies entirely on one side of $T_{\theta}$, hence escapes in the opposite direction. Suppose, then, that $l$ is not horizontal. Let $\Pi$ be any plane through $l$. $\Pi$ meets the $(x, y)$-plane in a line having some direction $\theta$. Look at the plane $z=\theta$. By varying II slightly, if necessary, we may assume that the intersection $l_{1}$ of II with that plane misses the $z$-axis, say by some distance $\varepsilon>0$. Since $l_{1}$ varies continuously with the choice of $\Pi$ through $l$, we can choose $\Pi^{\prime}$ sufficiently close to $\Pi$ so that the corresponding line $l^{\prime}$ (at height $\theta^{\prime}$ equal to its horizontal direction) lies at distance $>\varepsilon / 2$ from the $z$-axis, but so that the strip in the direction parallel to $l^{\prime}$ lies entirely within $\varepsilon / 2$ of the $z$-axis (i.e., has width $<\varepsilon$ ). Since the only lines that can "block" $\mathbf{P}$ ' in either direction are those parallel to $\Pi^{\prime}$, hence in the strip in question, it follows that $\Pi^{\prime}$ can escape, i.e., that $l$ is not in the convex hull of $\mathscr{L}$.

\section{Convex Connected Components}

In this section it is more convenient to work in the affine oriented Grassmannian, $\overline{\mathbf{G}}_{1, d}^{\prime}$, consisting of all oriented lines in $\mathbb{R}^{d}$. If $\mathscr{A}$ is a family of convex sets in $\mathbb{R}^{d}$, let $\mathscr{A}^{\mathscr{*}}$ represent the family of oriented $k$-flats meeting all the members of $\mathscr{A}$. Clearly, each unoriented $k$-transversal of $\mathscr{A}$ gives rise to a pair of oriented $k$-transversals of $\mathscr{A}$.

Let $\mathscr{A}$ be a finite family of pairwise disjoint compact convex point sets in $\mathbb{R}^{d}$ and let $\mathscr{A}^{\circledast}$ be its set of oriented line transversals. Each line in $\mathscr{A}^{\circledast}$ induces a linear ordering on the sets in $\mathscr{A}$ corresponding to the order in which the line meets the members of $\mathscr{A}$. Each transversal within a given connected component of $\mathscr{A}^{\circledast}$ induces 
the same linear ordering on $\mathscr{A}$. (This follows easily from the pairwise disjointness; for more details, and a generalization to $k$-transversals for $k>1$, see [3].) Thus each connected component of $\mathscr{A}^{\oplus}$ is associated with some linear ordering of $\mathscr{A}$. Similarly, each connected component of the space $\mathscr{A}^{*}$ of unoriented line transversals is associated with a pair of linear orderings of $\mathscr{A}$ (usually referred to as a geometric permutation of $\mathscr{A}$ [4].)

Two or more connected components of $\mathscr{A}^{\circledast}$ may be associated with the same linear ordering of $\mathscr{A}$. (For an example, see p. 174 of [3]). The exception is in $\mathbb{R}^{2}$, where components of $\mathscr{A}^{\circledast}$ are associated with distinct linear orderings, and (likewise) components of $\mathscr{A}^{*}$ with distinct pairs of linear orderings. More generally, if $\mathscr{A}$ is suitably separated, distinct components of the space of hyperplane transversals are associated with distinct order types (see [3]). A proof for unoriented hyperplane transversals in $\mathbb{R}^{d}$, which can easily be adapted to the oriented case, can be found in [5]. We include a proof here for the case of oriented lines in $\mathbb{R}^{2}$ for the convenience of the reader.

Lemma 1. Let $\mathscr{A}$ be a finite family of painwise disjoint compact convex point sets in $\mathbb{R}^{2}$. Each connected component of $\mathscr{A}^{\circledast}$ determines a distinct linear ordering of $\mathscr{A}$.

Proof. Suppose directed lines $l, l^{\prime} \in \mathscr{A}^{\circledast}$ meet the members of $\mathscr{A}$ in the same order $a_{1}, \ldots, a_{n}$. We show that $l$ can be moved continuously to $l^{\prime}$ through $\mathscr{A}^{\oplus}$.

If $\mathscr{A}$ consists of a single set, then $\mathscr{A}^{\circledast}$ is connected and the theorem is trivially true.

Assume $\mathscr{A}$ contains two or more sets. If $l$ is parallel to $l^{\prime}$, then it must have the same orientation as $l^{\prime}$. Then all the parallel lines between $l$ and $l^{\prime}$ with the same orientation as $l$ lie in $\mathscr{A}^{\circledast}$ and connect $l$ to $l^{\prime}$.

Suppose $l$ intersects $l^{\prime}$ at a point $p$. Lines $l$ and $l^{\prime}$ divide the plane into four quadrants labeled $1,2,3,4$ in clockwise order as in Fig. 1. For each set $a_{i} \in \mathscr{A}$, choose points $\alpha_{i} \in l \cap a_{i}$ and $\alpha_{i}^{\prime} \in l^{\prime} \cap a_{i}$. Each line segment $\overline{\alpha_{i} \alpha_{i}^{\prime}}$ lies in a single quadrant. (If a segment lies on one of the quadrant boundaries, assign it arbitrarily to one of the adjacent quadrants.)

Two or more line segments cannot lie in quadrants 2 and 4 , since $l$ and $l^{\prime}$ would meet these segments in different orders. If a single segment, $\overline{\alpha_{k} \alpha_{k}^{\prime}}$, is contained in these two quadrants, no endpoint $\alpha_{i}$ or $\alpha_{i}^{\prime}$ lies between $\alpha_{k}$ and $p$ or $\alpha_{k}^{\prime}$ and $p$. Rotate $l$ around $\alpha_{k}$ until it passes through $\alpha_{k}^{\prime}$ and then rotate it around $\alpha_{k}^{\prime}$ to $l^{\prime}$. If no segments are contained in quadrants 2 and 4 , rotate $l$ around $p$ to $l^{\prime}$.

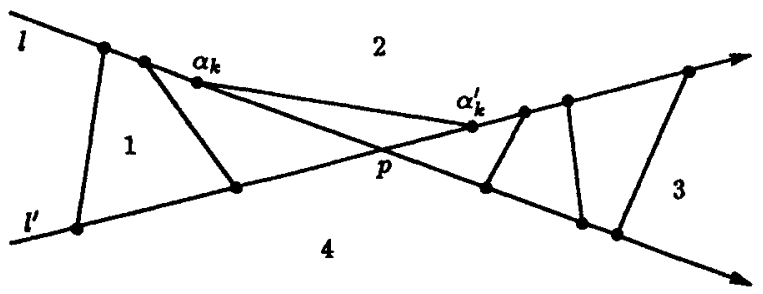

Fig. 1. Directed lines inducing the same linear ordering. 
Lemma 1 has a simple corollary for line transversals in any dimension.

Corollary 1. Let $\mathscr{A}$ be a finite family of painwise disjoint compact convex point sets in $\mathbb{R}^{d}$. If $l, l^{\prime} \in \mathscr{A}^{\circledast}$ meet the members of $\mathscr{A}$ in the same order and are coplanar, then $l$ and $l^{\prime}$ lie in the same connected component of $\mathscr{A}^{\circledast}$.

Proof. Assume that $l, l^{\prime} \in \mathscr{A}^{\circledast}$ generate the same ordering on $\mathscr{A}$ and that $l$ and $l^{\prime}$ both lie in a plane $\Pi$. Let $\mathscr{A}^{\prime}=\{\Pi \cap a \mid a \in \mathscr{A}\}$, considered as a family of subsets of $\Pi$. By Lemma $1, l$ and $l^{\prime}$ are connected through the space of transversals of $\mathscr{A}^{\prime}$. Since every transversal of $\mathscr{A}^{\prime}$ is a transversal of $\mathscr{A}$, it follows that $l$ and $l^{\prime}$ lie in the same connected component of $\mathscr{A}^{\circledast}$.

We refer to a vector $v$ as a positive combination of vectors $v_{1}, v_{2}, \ldots, v_{n}$ if $v=\sigma_{1} v_{1}+\sigma_{2} v_{2}+\cdots+\sigma_{n} v_{n}$ for real numbers $\sigma_{i} \geq 0$. The set of all positive combinations of finitely many vectors belonging to a set $V$ is the positive hull of $V$; it is a convex cone centered at the origin $O$.

As before, let be a finite family of pairwise disjoint compact convex point sets in $\mathbb{R}^{d}$. For each directed line $l$, let $v_{l}$ be the unit vector in the direction of $l$. For each connected component $\mathscr{L} \subseteq \mathscr{A}^{\circledast}$, define $C(\mathscr{L})$ to be the positive hull of the vectors $v_{l}, l \in \mathscr{L}$.

Lemma 2. Let $\mathscr{A}$ be a finite family of at least two pairwise disjoint compact convex point sets in $\mathbb{R}^{3}$ and let $\mathscr{L}$ be a connected component of $\mathscr{A}^{\circledast}$. A directed line $l \in \mathscr{A}^{\circledast}$ belongs to $\mathscr{L}$ if and only if $v_{l}$ belongs to $C(\mathscr{L})$.

Proof. If $l$ is in $\mathscr{L}$, then by definition $C(\mathscr{L})$ contains $v_{l}$. Assume that $l$ lies in $\mathscr{A}^{\circledast}$ but not in $\mathscr{L}$. We wish to show that $v_{1}$ does not lie in $C(\mathscr{L})$.

Let $\tilde{l}$ be some line in $\mathscr{L}$, and suppose first that $l$ and $\tilde{l}$ meet $\mathscr{A}$ in different orders. There is some pair of sets $a, b \in \mathscr{A}$ such that $l$ meets $a$ before $b$ and $\tilde{l}$ meets $b$ before $a$. Let $h$ be a plane separating $a$ from $b$. Every line in $\mathscr{L}$ must point toward the half-space bounded by $h$ containing $a$, while line $l$ points toward the half-space bounded by $h$ containing $b$. Thus $v_{l}$ cannot be a positive combination of the vectors $v_{l^{\prime}}, l^{\prime} \in \mathscr{L}$.

Now suppose $l$ meets the members of $\mathscr{A}$ in the same order that $\tilde{l}$ does. Let $I I$ be a plane perpendicular to $l$, and denote by $\Pi(a), \Pi\left(l^{\prime}\right)$, and $\Pi(v)$ the orthogonal projection of any set $a \in \mathscr{A}$, any line $l^{\prime} \in \mathbb{R}^{3}$, and any vector $v \in \mathbb{R}^{3}$ onto $\Pi$. If $v_{l}$ is a positive combination of vectors $v_{l^{\prime}}, l^{\prime} \in \mathscr{L}$, then the origin, $O$, is in the convex hull of $\Pi\left(v_{l^{\prime}}\right), l^{\prime} \in \mathscr{L}$.

Let $\kappa=\bigcap_{\alpha \in \infty} \Pi(a)$. (See Fig. 2.) Notice that if a line $l^{\prime}$ is parallel to $l$, then $l^{\prime}$ is in $\mathscr{A}^{\oplus}$ if and only if $l^{\prime} \cap \Pi$ lies in $\kappa$. Each directed line of $\mathscr{A}^{*}$ parallel to $l$ and oriented in the same direction is clearly in the same connected component of $\mathscr{A}^{\circledast}$ as l. By Corollary 1 , no such line intersects a line of $\mathscr{L}$. Thus $\Pi\left(l^{\prime}\right)$, for each line $l^{\prime} \in \mathscr{L}$, misses $\kappa$.

Without loss of generality, assume that $\kappa$ lies to the left to $\Pi(\tilde{l})$. Since $\Pi\left(l^{\prime}\right)$ is a directed line that misses $\kappa$ for every $l^{\prime} \in \mathscr{L}$ and $\mathscr{L}$ is connected, $\kappa$ must be to the left of $\Pi\left(l^{\prime}\right)$ for every $l^{\prime} \in \mathscr{L}$. Let $t$ be the translate of $\Pi(\tilde{l})$ supporting $\kappa$ on its left. 


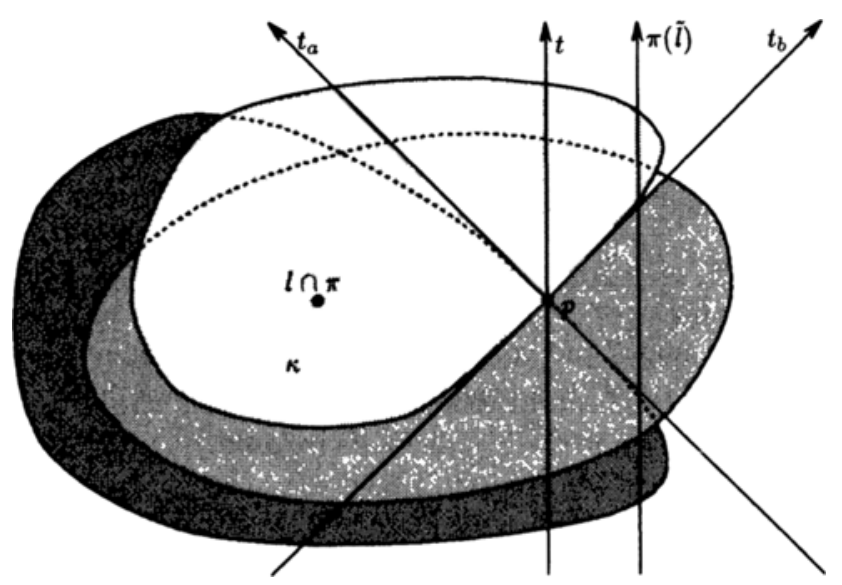

Fig. 2. Projection of sets onto plane II.

Line $t$ does not support any $\Pi(a), a \in \mathscr{A}$, or else $\tilde{l}$ would not intersect $a$. Thus $t \cap \kappa$ consists of only a single point $p$, since otherwise some line strictly between $\tilde{l}$ and $t$ would already support $\kappa$. Let $t^{+}$be the open half-plane in $\Pi$ bounded by $t$ not containing $\kappa$. By Helly's theorem, there are two distinct point sets $\Pi(a), \Pi(b)$, with $a, b \in \mathscr{A}$, such that $\Pi(a) \cap \Pi(b) \cap t^{+}=\emptyset$.

Let $t_{a}$ and $t_{b}$ be supporting lines to $\operatorname{II}(a)$ and $\Pi(b)$ at $p$. Orient $t_{a}$ and $t_{b}$ in directions $v_{a}$ and $v_{b}$, respectively, so that $\Pi\left(v_{i}\right)$ lies in the open cone between $v_{a}$ and $v_{b}$. For each $l^{\prime} \in \mathscr{L}$, the vector $\Pi\left(v_{l}\right)$ is not a positive scalar multiple of $v_{a}$ or $v_{b}$ or else $l^{\prime}$ would not intersect $a$ or $b$. Since $\mathscr{L}$ is connected, all the vectors $\operatorname{II}\left(v_{l^{\prime}}\right)$, $l^{\prime} \in \mathscr{L}$, also lie in the open cone between $v_{a}$ and $v_{b}$. Thus 0 is not a convex combination of $\Pi\left(v_{l}\right), l^{\prime} \in \mathscr{L}$, and so $v_{l}$ is not in the positive hull of $v_{l^{\prime}}, l^{\prime} \in \mathscr{L}$.

The following simple lemma holds for $k$-transversals in $\mathbb{R}^{d}$ for any $k$ and $d$.

Lemma 3. Let $\mathscr{A}$ be a nonempty family of convex point sets in $\mathbb{R}^{d}$ and let $\mathscr{A}^{*}$ and $\mathscr{A}^{\circledast}$ represent the sets of unoriented and oriented $k$-transversals of $\mathscr{A}$, respectively. If every point set in $\mathscr{A}$ is compact, then so are $\mathscr{A}^{*}$ and $\mathscr{A}^{\oplus}$.

Proof. For each compact convex set $a \in \mathscr{A}$, the set of (oriented) $k$-transversals of $a$ is compact. $\mathscr{A}^{*}\left(\mathscr{A}^{(*)}\right)$ is the intersection of these compact sets of (oriented) $k$-transversals and so is also compact.

We return to lines in $\mathbb{R}^{3}$ where $\mathscr{A}^{\circledast}$ is the set of oriented line transversals of $\mathscr{A}$. Given a set $\mathscr{L}$ of directed lines, let us denote the set of lines with reverse orientation by $\mathscr{L}^{\text {rev }}$. If $\mathscr{L}$ is a connected component of $\mathscr{A}^{\circledast}$, then clearly $\mathscr{L}^{\text {rev }}$ is also,

Theorem 1. Let $\mathscr{A}$ be a finite family of painwise disjoint compact convex point sets in $\mathbb{R}^{3}$. If $\mathscr{L}$ is a connected component of $\mathscr{A}^{\circledast}$, then $\mathscr{L} \cup \mathscr{L}^{\mathrm{rev}}$ is convex. Moreover, 
$\mathscr{L} \cup \mathscr{L}^{\mathrm{rev}}$ is itself the space of oriented line transversals of some finite family of pairwise disjoint compact convex point sets.

Proof. If $\mathscr{A}$ consists of a single convex set, then $\mathscr{A}^{\circledast}$ has just one connected component and the theorem is trivially true. We therefore assume that $\mathscr{A}$ consists of more than one convex set.

Let $\mathscr{L}$ be a connected component of $\mathscr{A}^{\circledast}$. Let $h$ be some plane strictly separating a pair of sets in $\mathscr{A}$ translated to pass through $O$. The vectors $v_{l}, l \in \mathscr{L}$, all lie in one of the open half-spaces, say $h^{+}$, bounded by $h$. Thus $C(\mathscr{L})$ lies in $h^{+}$as well.

By Lemma 3 , since $\mathscr{A}$ is compact, so is $\mathscr{A}^{\circledast}$. Hence so are the sets $\mathscr{L}$ and $\mathscr{A}^{\circledast} \backslash \mathscr{L}$, and the convex cone $C(\mathscr{L})$ is closed. By Lemma 2, if $l \in \mathscr{A} \mathscr{H}^{\circledast} \backslash \mathscr{L}$, then $v_{l}$ does not lie in $C(\mathscr{L})$. Hence, by compactness, there is an angle $\alpha>0$ such that the angle between $v_{l}$, for any $l \in \mathscr{L}^{\circledast} \backslash \mathscr{L}$, and any vector $v \in C(\mathscr{L})$ is always greater than $\alpha$. We can therefore enlarge $C(\mathscr{L})$ slightly to form a new closed convex cone $C^{\prime}$ with vertex $O$ such that $C(\mathscr{L})$ lies in the interior of $C^{\prime}, C^{\prime} \subset h^{*}$, and $v_{l}$ does not lie in $C^{\prime}$ for any $l \in \mathscr{A}^{\circledast} \backslash \mathscr{L}$.

Consider a directed line $l \in \mathscr{A}^{\oplus}$. Line $l$ belongs to $\mathscr{L} \cup \mathscr{L}^{\text {rev }}$ if and only if $v_{l}$ or $-v_{l}$ lies in the interior of $C^{\prime}$. Hence $l \in \mathscr{L} \cup \mathscr{L}^{\text {rev }}$ if and only if $l \cap C^{\prime}$ is unbounded. By compactness, there is a ball $B$ centered at $O$ such that $C \cap l$ is contained in $B$ for every $l$ in $\mathscr{A}^{\circledast} \backslash\left(\mathscr{L} \cup \mathscr{L}^{\mathrm{rev}}\right)$. Similarly by compactness, there is a ball $B^{\prime}$ centered at $O$ such that $C \cap l$ meets $B^{\prime}$ for every $l \in \mathscr{L}$. Finally, let $B^{\prime \prime}$ be a ball centered at $O$ containing all the sets $a \in \mathscr{A}$. Call the largest of these three balls $B^{\prime \prime \prime}$. Translate the plane $h$ to a plane $h^{\prime}$ that intersects the cone $C^{\prime}$ but not the ball $B^{\prime \prime \prime}$. Let $c=C^{\prime} \cap h^{\prime}$, and notice that since $C^{\prime} \subset h^{+}$was closed, $c$ is compact. Then every line in $\mathscr{L} \cup \mathscr{L}^{\text {rev }}$ intersects $c$, while no line in $\mathscr{A}^{\circledast} \backslash\left(\mathscr{L} \cup \mathscr{L}^{\text {rev }}\right)$ does. Thus $\mathscr{A} \cup\{c\}$ consists of pairwise disjoint compact convex sets, and we have $\mathscr{L} \cup \mathscr{L}^{\text {rev }}=$ $(\mathscr{A} \cup\{c\})^{\circledast}$.

Our desired result then follows as a corollary.

Corollary 2. Let $\mathscr{A}$ be a finite family of painwise disjoint compact convex point sets in $\mathbb{R}^{3}$. If $\mathscr{L}$ is a connected component of $\mathscr{A}^{*}$, then $\mathscr{L}$ is convex. Moreover, $\mathscr{L}$ is itself the space of oriented line transversals of some finite family of painwise disjoint compact convex point sets.

Proof. Again, we may assume without loss of generality that $\mathscr{A}$ consists of at least two convex sets. Since the sets in $\mathscr{A}$ are disjoint, $\mathscr{L}$ gives rise to two connected components of $\mathscr{A}^{\circledast}$ coming from the two orientations of lines in $\mathscr{L}$. By Theorem 1 , there is a compact convex set $c$ disjoint from each of the sets in $\mathscr{A}$ such that the components together constitute the space of directed line transversals of $\mathscr{A} \cup\{c\}$. Thus $\mathscr{L}$ is the space of undirected line transversals of $\mathscr{A} \cup\{c\}$.

\section{References}

1. Eckhoff, J. Helly, Radon, and Carathéodory type theorems. In Handbook of Convex Geometry, P. M. Gruber and J. M. Wills, eds. North-Holland, Amsterdam, 1993, pp. 389 448. 
2. Goodman, J. E., and Pollack, R. Foundations of a theory of convexity on affine Grassmarn manifolds. Mathematika (to appear).

3. Goodman, J. E., Pollack, R., and Wenger, R. Geometric transversal theory, In New Trends in Discrete and Computational Geometry, J. Pach, ed. Springer-Verlag, Heidelberg, 1993, pp. 163-198.

4. Katchalski, M., Lewis, T., and Zaks, J. Geometric permutations for convex sets. Discrete Math. 54 (1985), 271-284.

5. Wenger, R. Geometric permutations and connected components. Tech. Report TR-90-50, DIMACS, 1990.

Received January 3, 1994, and in revised form April 27, 1994. 